\title{
Prethermalization without Temperature
}

\author{
David J. Luitz $\odot,{ }^{1}$ Roderich Moessner, ${ }^{1}$ S. L. Sondhi, ${ }^{2}$ and Vedika Khemani ${ }^{3,4}$ \\ ${ }^{1}$ Max-Planck-Institut für Physik komplexer Systeme, 01187 Dresden, Germany \\ ${ }^{2}$ Department of Physics, Princeton University, Princeton, New Jersey 08544, USA \\ ${ }^{3}$ Department of Physics, Harvard University, Cambridge, Massachusetts 02138, USA \\ ${ }^{4}$ Department of Physics, Stanford University, Stanford, California 94305, USA
}

(Received 16 October 2019; revised manuscript received 25 January 2020; accepted 6 March 2020; published 29 May 2020)

\begin{abstract}
While a clean, driven system generically absorbs energy until it reaches "infinite temperature," it may do so very slowly exhibiting what is known as a prethermal regime. Here, we show that the emergence of an additional approximately conserved quantity in a periodically driven (Floquet) system can give rise to an analogous long-lived regime. This can allow for nontrivial dynamics, even from initial states that are at a high or infinite temperature with respect to an effective Hamiltonian governing the prethermal dynamics. We present concrete settings with such a prethermal regime, one with a period-doubled (time-crystalline) response. We also present a direct diagnostic to distinguish this prethermal phenomenon from its infinitely long-lived many-body localized cousin. We apply these insights to a model of the recent NMR experiments by Rovny et al. [Phys. Rev. Lett. 120, 180603 (2018)] which, intriguingly, detected signatures of a Floquet time crystal in a clean three-dimensional material. We show that a mild but subtle variation of their driving protocol can increase the lifetime of the time-crystalline signal by orders of magnitude.
\end{abstract}

DOI: 10.1103/PhysRevX.10.021046

\section{INTRODUCTION}

The study of quantum systems out of equilibrium has led to the identification of fundamentally new phenomena, such as the discrete time crystal (DTC) in periodically driven (Floquet) systems [1-7]. In a generic many-body system, periodic driving leads to heating to a featureless "infinite temperature" state, appropriate to maximizing entropy in a system with no conservation laws [8-10]. The only known generic mechanism for avoiding this heating $[8,10-13]$-in the asymptotic limit of large systems and late times-relies on the phenomenon of many-body localization (MBL) in disordered, interacting systems [14-23]. This permits the existence of nontrivial MBL Floquet phases [1], the DTC being a paradigmatic example which displays a novel form of long-range spatiotemporal order-breaking both the discrete time-translation symmetry of the periodic drive and an emergent (spatial) Ising symmetry [1,3,5,24].

Many-body localization requires a number of idealized conditions (for example, perfect environmental isolation and short-ranged interactions) that may not always be

Published by the American Physical Society under the terms of the Creative Commons Attribution 4.0 International license. Further distribution of this work must maintain attribution to the author(s) and the published article's title, journal citation, and DOI. Open access publication funded by the Max Planck Society.

\author{
Subject Areas: Condensed Matter Physics, \\ Statistical Physics, \\ Strongly Correlated Materials
}

realized in a given experimental setup. Nevertheless, even absent MBL, it was shown that the heating time can be made exponentially large in some dimensionless system parameters, $t_{h} \sim O(\exp (\omega / J))$, when the driving frequency $\omega$ is large compared to the local energy scales in the system, $\sim J$ [25-29]. Intuitively, absorbing one "quantum" of energy $\omega$ from the drive requires the rearrangement of many local degrees of freedom with energy scales $J$, which is a high-order process leading to parametrically slow heating.

In the "prethermal" regime prior to heating, $t<t_{h}$, the system can display nontrivial dynamics and is well described by a (quasilocal) time-independent "effective Hamiltonian" $H_{\text {eff }}$ that captures the dynamics of the system out to an exponentially long time [26-29]. Building on this, it was shown in Ref [30] that a DTC can be realized for an extended prethermal regime, even absent MBL, if one arranges for (a slightly generalized) $H_{\text {eff }}$ to additionally display an emergent Ising symmetry, with a spontaneous Ising symmetry-breaking transition at some temperature $T_{c}$. Then, upon starting from a symmetry-broken initial state at a low temperature below $T_{c}$, the system can display oscillations of the Ising order parameter at twice the driving period. At late times, the system eventually heats to infinite temperature and $H_{\text {eff }}$ ceases to be a good description. We will refer to such prethermal time crystals that rely on spontaneous symmetry breaking (SSB) as "prethermal SSB DTCs." 
Intriguingly, a recent NMR experiment on a clean, periodically driven three-dimensional crystal observed signatures of time crystallinity, despite being far from any MBL regime [31,32]. The experiment measured the global magnetization of the sample, and observed period-doubled oscillations for the duration of the experimental coherence time (about a 100 driving periods). Despite the almost complete lack of disorder, the observed signal was very similar to that observed in two earlier experiments, on disordered nitrogen-vacancy centers [33] and trapped ions [34], that were closer in spirit to MBL TCs due to slow disorder-impeded thermalization [35].

A natural conjecture is that the clean NMR experiment may be seeing a prethermal SSB DTC as in Ref. [30]. However, the experiment prepares a weakly polarized initial state that is at an extremely high temperature (vastly in excess of the strength of the dipolar interactions in the crystal). This does not satisfy the requirement in [30] for starting with a symmetry-broken initial state at a low temperature with respect to $H_{\text {eff }}$.

Thus, the NMR results do not fit into any existing framework of Floquet MBL (or prethermal) order, and call for a new theory. We identify the emergence of a long-lived approximately conserved quantity as the crucial missing ingredient. The existence of this conserved quantity stabilizes the time-crystalline behavior and provides a prethermal window via a long timescale on which this conservation law is eventually destroyed. This conservation law may or may not be accompanied by the presence of approximate long-lived energy conservation (i.e., the existence of a local time-independent $H_{\text {eff }}$ ) in previously identified prethermal phenomena, thereby extending these qualitatively. We also emphasize that the existence of this conservation law does not, in turn, require any (conventional) spontaneous symmetry breaking.

To make contact with the experiment, we arrange for the emergence of a long-lived U(1) symmetry, that is approximately the total spin (or global magnetization $M$ ) along the $z$ direction. We primarily focus on cases where there is also long-lived energy conservation and hence an $H_{\text {eff }}$. Here, one can show that dynamics from initial states at infinite temperature but nonzero magnetization density can nevertheless show nontrivial dynamics [such as long-lived oscillations of $M(t)$ ] for a long period of time, thereby allowing for the apparently oxymoronic notion of prethermalization without temperature.

One of the insights deriving from our analysis is that a prethermal DTC signal is most stable for parameter values which may not have been a priori obvious. In particular, a well-known route to realizing an approximate U(1) symmetry in a time-independent system is to apply a large magnetic field in the, say, $z$ direction [27]. However, as we discuss below, this is not as straightforward in some natural Floquet settings since the stroboscopic nature of the Floquet unitary does not allow for the accumulation of arbitrarily large phases.
The crispest mechanism for realizing the physics we have in mind entails engineering the desired emergent symmetry to leading order in $H_{\text {eff }}$, with residual symmetrybreaking perturbations arising only at higher orders in a small parameter $\epsilon / \omega$. In more detail, a fundamental object of interest in a Floquet system is the time-evolution operator over one driving period $T$, defined as $U(T)=$ $\mathcal{T} e^{-i \int_{0}^{t} d t H(t)}$. This can be used to formally define a (nonunique) "Floquet Hamiltonian" $\hat{H}_{F}$ via $\hat{U}(T)=$ $\mathcal{T} e^{-i \int_{0}^{T} d t \hat{H}(t) / \hbar} \equiv e^{-i \hat{H}_{F} T / \hbar}$, where the operator $\hat{H}_{F}$ is generally highly nonlocal in a many-body system. When $\omega$ is large compared to the local energy scales of the problem, one can perform a high-frequency asymptotic expansion for $\hat{H}_{F}$ in powers of $1 / \omega, \hat{H}_{F}=\sum_{n}(1 / \omega)^{n} \hat{\mathcal{H}}_{F}^{(n)}$; the leadingorder term $\hat{\mathcal{H}}_{F}^{(0)}$ is the time-averaged Hamiltonian, while higher-order terms are progressively longer ranged and contribute significantly to the dynamics only at correspondingly later times. While ultimately divergent, this expansion looks convergent out to some optimal order $n_{\text {opt }}=$ $O(\omega / J)$. Truncating the expansion at this order yields $H_{\text {eff }}$ which is an exponentially accurate approximation to the Floquet time evolution $\hat{U}(T)$, thereby setting the rate of heating to be exponentially small [26-29]. If $\hat{\mathcal{H}}_{F}^{(0)}$ has the desired symmetry, with violations coming in at higher orders with strength $(\epsilon / \omega)^{n}$ (where $\epsilon$ is an independently chosen small parameter), then the timescale on which the symmetry is destroyed can be made parametrically large for small $\epsilon$ and large $\omega$.

In sum, our work (i) widens the scope of Floquet prethermalization, (ii) expands the toolkit for using the Floquet system to generate dynamics with novel drives and symmetries, and (iii) sheds light on the mystery of the NMR time-crystal experiment. In particular, we also predict that a slight and straightforward modification of the original experimental NMR protocol $[31,32]$ - a judicious choice of an optimal magnetic field driving protocol-will exponentially enhance the many-body lifetime of the observed DTC.

The rest of this manuscript is structured as follows. In Sec. II, we present the drive studied in the NMR DTC experiment, and introduce a family of short-range interacting one-dimensional spin $1 / 2$ models inspired by the experiment as model systems to provide evidence confirming our picture. We then analyze the regimes of thermalization for our model drives in Sec. III, showing how one can engineer a long-lived approximate emergent $\mathrm{U}(1)$ conservation that can show nontrivial magnetization dynamics even at infinite temperature and enhance the lifetime of the DTC signal observed in the NMR experiment. Section IV provides concrete signatures for distinguishing between the different MBL and prethermal regimes in experiment, while Sec. V concludes with a summary and outlook. 


\section{NMR FLOQUET DRIVE}

In order to keep this work self-contained, we briefly summarize the pertinent details of the NMR DTC experiment of Refs. [31,32]. A standard NMR setup entails nuclear spins $\mathbf{I}_{i}$ located on sites $i$ of a crystalline lattice, interacting via dipolar interactions, $J_{i j} \sim \mu_{0} \gamma_{i} \gamma_{j} /$ $\left(4 \pi\left|\mathbf{r}_{i j}\right|^{3}\right)\left[\mathbf{I}_{i} \cdot \mathbf{I}_{j}-3\left(\mathbf{I}_{i} \cdot \hat{\mathbf{r}}_{i j}\right)\left(\mathbf{I}_{j} \cdot \hat{\mathbf{r}}_{i j}\right)\right]$ for spins separated by the lattice vector $\mathbf{r}_{i j}$, where $\mu_{0}$ is the vacuum permeability, and $\gamma_{i}$ and $\gamma_{j}$ are the nuclear gyromagnetic ratios of the two spins. In the NMR DTC experiment [31], the spins are furnished by spin- $1 / 2{ }^{31} P$ nuclei in ammonium dihydrogen phosphate, and are arranged in a three-dimensional crystalline lattice. As is typical of NMR experiments, the setup is subject to a strong magnetic field oriented along the $z$ direction (by convention). The Zeeman splitting of the nuclear spins from this applied field is several orders of magnitude larger than the strength of the dipolar interactions, and the Zeeman field leads to a very fast precession of all transverse components of the nuclear spins. Then, in the rotating frame of this large applied field, one can define a so-called "secular" Hamiltonian which takes a "XXZ" form for interactions between spins of the same type [31]:

$$
\begin{aligned}
H_{\text {secular }}= & \sum_{i, j} \frac{\mu_{0} \gamma_{i} \gamma_{j}}{4 \pi\left|\mathbf{r}_{i j}\right|^{3}} \frac{1}{2}\left[3 \cos ^{2}\left(\theta_{i j}\right)-1\right]\left(\mathbf{I}_{i} \cdot \mathbf{I}_{j}-3 I_{i}^{z} I_{j}^{z}\right) \\
& +h \sum_{i} I_{i}^{z}+\cdots,
\end{aligned}
$$

where $\theta_{i j}$ is the angle between the internuclear vector $\mathbf{r}_{i j}$ and the $z$ axis (defined by the static external field), $I_{i}^{z}$ refers to the $z$ component of the spin $\mathbf{I}_{i}$, and $\cdots$ refers to other couplings between other types of nuclear spins and higherorder terms. Note that the secular Hamiltonian conserves the total $z$ component of the magnetization, $M=\sum_{i} I_{i}^{z}$.

The DTC experiment [31,32] prepares a weakly magnetized mixed initial state at high temperature. The spins interact via $H_{\text {secular }}$ for a time period $T$ and are then periodically subject to a near-perfect $\pi$ pulse that globally flips all spins, with a deviation $\epsilon$. Despite the systematic deviation in the rotation angle, the experiment observes a period-doubled signal locked at a frequency $\omega / 2$ for strongenough interactions $J$, one characteristic signature of timecrystalline order [5]. For weaker interactions, the system crosses over to a regime with "beating" at a frequency that tracks $\epsilon$ instead of robust period doubling - thereby crossing over from a regime with a time-crystalline signature to one without.

For concreteness, we will work with a tractable minimal model inspired by the NMR drive in which the essential ingredients for producing DTC behavior via U(1) prethermalization are manifest. We study a driven onedimensional system of spin degrees of freedom on sites $i$, $S_{i}^{\alpha}=\frac{1}{2} \sigma_{i}^{\alpha}$ where $\alpha=x, y, z$, and $\sigma^{\alpha}$ are Pauli spin $1 / 2$ matrices. The drive consists of three elements. The first are $X X Z$-type nearest and (integrability-breaking) nextnearest-neighbor interactions of respective strengths $J, J^{\prime}$; the second a uniform magnetic field in the $z$ direction, $h S_{\text {tot }}^{z}$; and the third a periodically applied global spin rotation by an angle $\theta$ about the $x$ axis, generated by $P_{\theta}^{x}$. Our choice to work in one dimension with truncated-range interactions is for numerical tractability; our conceptual framework is equally applicable to higher dimension and longer-range interactions.

The model drive is

$$
\begin{aligned}
P_{\theta}^{x}= & e^{-i \theta S_{\mathrm{tot}}^{x},} \\
H_{c}= & J \sum_{i=1}^{L-1}\left(S_{i}^{x} S_{i+1}^{x}+S_{i}^{y} S_{i+1}^{y}-2 S_{i}^{z} S_{i+1}^{z}\right) \\
& +J^{\prime} \sum_{i=1}^{L-2}\left(S_{i}^{x} S_{i+2}^{x}+S_{i}^{y} S_{i+2}^{y}-2 S_{i}^{z} S_{i+2}^{z}\right),
\end{aligned}
$$

with $J=1, J^{\prime}=0.5$. The resulting Floquet unitary, which is the stroboscopic time evolution operator over one period, is given by

$$
U(T)=P_{\theta}^{x} e^{-i T_{1}\left(H_{c}+h S_{\mathrm{tot}}^{z}\right)}=P_{\theta}^{x} e^{-i h T_{1} S_{\mathrm{tot}}^{z}} e^{-i T_{1} H_{c}},
$$

where the second line follows from the first because $\left[H_{c}, S_{\mathrm{tot}}^{z}\right]=0$ justifies the separation of exponentials. In what follows, we vary the period $T_{1}$ and field $h$, while considering small, $\theta=0+\epsilon$, and nearly maximal, $\theta=$ $\pi+\epsilon$ (" $\pi$ pulse"), spin rotation angles. These are detuned by a small amount $\epsilon=0.1$, unless otherwise stated, to address the stability of the phenomena we discuss. The exact $\pi$ pulse, $P_{\pi}^{x} \sim \prod_{i} \sigma_{i}^{x}$, enacts a perfect flip of all spins in the $z$ basis. For $\theta=\pi+\epsilon$, the flip has a systematic deviation, as in NMR experiment.

Let us discuss some salient features of this drive.

First, for $\epsilon=0$, the evolution can be identified with that of a static Hamiltonian with perfect $U(1)$ symmetry. This is trivially true when $\theta=0$, in which case the problem reduces to an undriven one, $U(T)=e^{-i T_{1}\left(H_{c}+h S_{\mathrm{tot}}^{z}\right)}$. For the "flipped" case with spin rotation angle $\theta=\pi$, this is still true if the two period unitary is considered, $U(T)^{2}=$ $e^{-i 2 T_{1} H_{c}}$, which follows from the fact that $\left[H_{c}, P_{\pi}^{x}\right]=0$ and $\left\{S_{\text {tot }}^{z}, P_{\pi}^{x}\right\}=0$. In the latter case, the U(1) symmetry can be used to achieve perfect period doubling (or "time-crystalline") dynamics with the global magnetization flipping every period: $M(n T)=(-1)^{n} M(0)$, where $M=\sum_{i} \sigma_{i}^{z}, n$ is an integer, and $M(n T)=$ $U^{\dagger}(n T) M U(n T)$. Again, this follows simply because $U(2 T)$ commutes with $M$ while $U(T)$ anticommutes with $M$ due to the action of the $\pi$ flip, and does not rely on symmetry breaking.

On the other hand, for any nonzero $\epsilon$, the system is genuinely driven and will eventually approach the infinite temperature ensemble $\rho \propto \mathbb{I}$. The challenge is thus to generate long timescales, $t_{h}$ and $t_{m}$, both for the 
approximate nonconservation of energy and the approximate nonconservation of $S_{\text {tot }}^{z}$, respectively.

Second, note that the second line of Eq. (3) implies that $h T_{1}$ is only defined modulo $2 \pi$, and hence cannot be made arbitrarily large for this drive: there is no simple high-field limit. We will show that the dynamics in this system can be explained via an approximate long-lived conservation of $S_{\text {tot }}^{z}$. However, the approximate conservation of $S_{\text {tot }}^{z}$ the central emergent feature-is not due to a large field $h$. Instead, it is the smallness of the detuning $\epsilon$ of the global spin rotation, which controls both the strength of driving and the strength of the $S_{\text {tot }}^{z}$ violation. We emphasize that while a large magnetic field is used in the NMR setup to obtain an interaction Hamiltonian $H_{c}$ that conserves $S_{\text {tot }}^{z}$ within the secular approximation, the periodic drive as a whole comprises both the interaction Hamiltonian (with the field) and the global spin rotation-and there is no simple large field limit to obtain $S_{\text {tot }}^{z}$ conservation for $U(T)$ as a whole.

Third, in the low-frequency regime when $\omega=2 \pi / T_{1} \ll$ $\{J, h\}$, the experiment finds that the envelope of $M(n T)$ decays exponentially in time so there is no long-lived signal. In this regime, there is no quasiconservation of energy and $|M(t)| \sim e^{-t / t_{d}}$, with a decay time $t_{d} \sim 1 / \epsilon^{2}$. This is the "dephasing regime" [36]. The basic picture is that the operator $S_{\text {tot }}^{z}$ gets "rotated away" from the $z$ axis by an angle $\epsilon$ every drive cycle under the action of $P_{\epsilon}^{x}$. The component of the polarization in the $X Y$ plane gets dephased under the action of $H_{c}$, while the component parallel to $z$ remains conserved while $H_{c}$ acts. This gives a decay $M(n) \sim \cos (\epsilon)^{n}$, consistent with the observed exponential decay upon expanding in small $\epsilon$. In the next section, we will work instead in the high-frequency limit which allows us to enter a prethermal regime.

\section{REGIMES OF THERMALIZATION}

In this section, we discuss various regimes of thermalization for the model drive in Eq. (3) in the high-frequency regime $\omega \gg\{J, h\}$. The demonstration of a long-lived U(1)-stabilized DTC signal proceeds in several steps. Our starting point is an analysis of the NMR experiment with its period-doubled response [Case 1, Fig. 1(a)]. We then demonstrate how to extend its lifetime dramatically into a bona fide prethermal signal by adjusting the drive parameters. This proceeds in two steps. We first arrange for the appearance of a prethermal regime by adjusting the drive [Case 2, Fig. 1(b)] to yield an effective Hamiltonian with emergent approximate $\mathrm{U}(1)$ conservation, but no period doubling. The second step [Case 3, Fig. 1(c)] recreates the period-doubled DTC response, now with a parametrically longer lifetime, by adjusting the applied Zeeman field. The resulting dramatic increase in lifetime is a consequence of the effective Hamiltonian, at leading order, being perfectly $\mathrm{U}(1)$ symmetric.

As discussed previously, the high-frequency regime $\omega \gg\{J, h, \theta\}$ allows one to define a quasilocal timeindependent effective Hamiltonian associated with the quasiconservation of energy [26-29]. The dynamics are well approximated by $H_{\text {eff }}$ up to the "heating time"

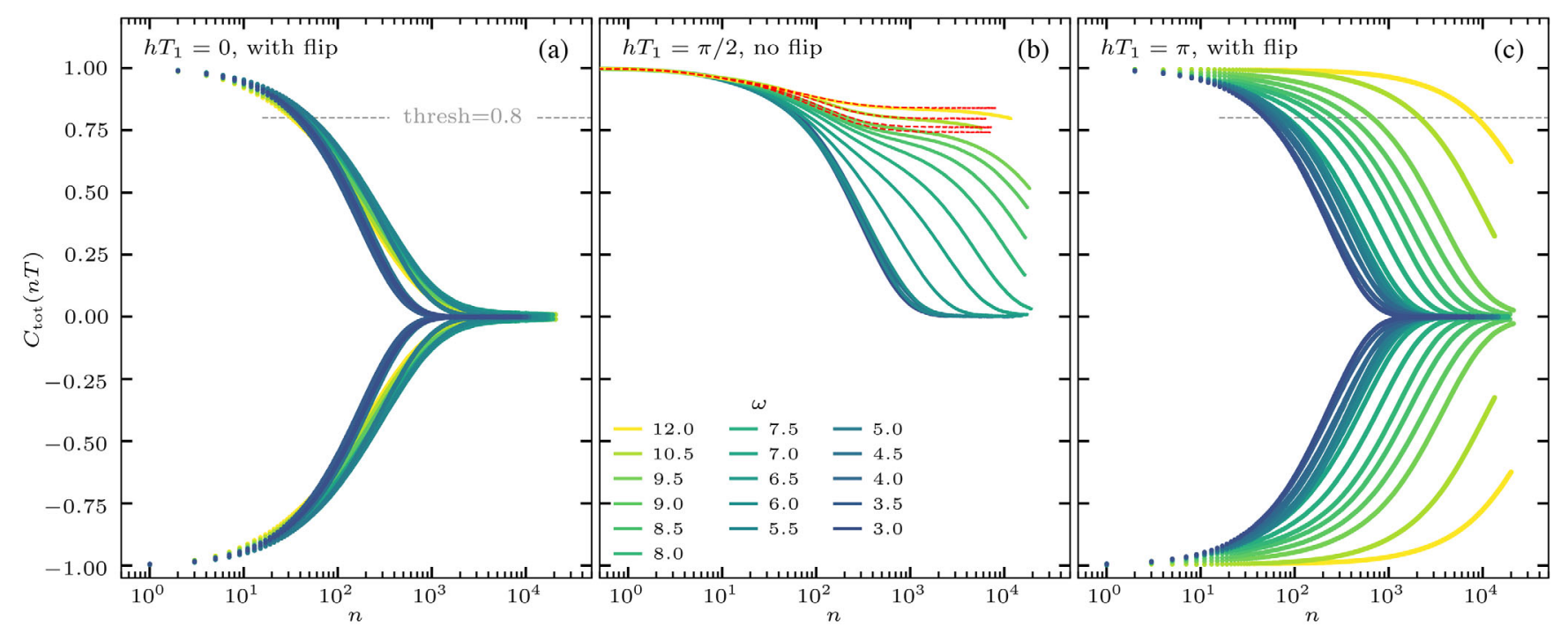

FIG. 1. Survival of the total magnetization $C_{\text {tot }}(n T)$ [defined in Eq. (6)], stroboscopically observed, in a chain of length $L=20$ under the NMR Floquet drive for different driving frequencies $\omega=2 \pi / T_{1}$ (3). The detuning of the spin rotation is $\epsilon=0.1$. (a) Without a field $(h=0)$ and with an approximate global flip $P_{\theta}^{x}, \theta=\pi+\epsilon$, corresponding to the presumptive parameters of the NMR experiment, the magnetization dies off quickly with little dependence on the driving frequency. (b) At half the maximum field, $h=\pi /\left(2 T_{1}\right)$, and with $\theta=0+\epsilon$, the survival of the magnetization is enhanced. Dashed lines show the evolution with the time-averaged leading-order effective Hamiltonian. (c) With the maximal field $h=\pi / T_{1}$ and with an approximate global spin flip $\theta=\pi+\epsilon$, we observe a prethermal time-crystalline signal with dramatically enhanced lifetime, by more than $100 \times$ compared to (a). The lifetime shows an exponential dependence on driving frequency (Fig. 2), a hallmark of prethermalization. 
$t_{h} \sim e^{\omega / J}$. We note that the existence of this prethermal regime requires $\omega$ to be much bigger than all local energy scales in the time-dependent Hamiltonian, Eq. (2). However, this is not strictly true when $\theta=\pi+\epsilon$, which is the regime in which one gets a nontrivial DTC phase. This issue is also encountered when considering the existence of Floquet MBL in the DTC phase, and the resolution is that one needs to eliminate the large frequency effect of the $\pi$ pulse first, which is conveniently done by either working in a "toggling" frame which rotates by $P_{\pi}^{x}$ each time a global rotation is applied or, equivalently, by considering the time evolution over two periods:

$$
\begin{aligned}
U(2 T) & =P_{\pi}^{x} P_{\epsilon}^{x} e^{-i h T_{1} S_{\mathrm{tot}}^{z}} e^{-i T_{1} H_{c}} P_{\pi}^{x} P_{\epsilon}^{x} e^{-i h T_{1} S_{\mathrm{tot}}^{z}} e^{-i T_{1} H_{c}} \\
& =P_{\epsilon}^{x} e^{+i h T_{1} S_{\mathrm{tot}}^{z}} e^{-i T_{1} H_{c}} P_{\epsilon}^{x} e^{-i h T_{1} S_{\mathrm{tot}}^{z}} e^{-i T_{1} H_{c}},
\end{aligned}
$$

where we have used $\left[H_{c}, P_{\pi}^{x}\right]=0$ and $\left\{S_{\text {tot }}^{z}, P_{\pi}^{x}\right\}=0$ to eliminate the large $\theta$ field. Then, one can define an effective Hamiltonian which, to leading order in $1 / \omega$, is a time average:

$$
\begin{aligned}
& H_{\text {eff }}=\hat{\mathcal{H}}_{F}^{(0)}+O(1 / \omega) \\
& \hat{\mathcal{H}}_{F}^{(0)} \propto \frac{T_{1}}{T_{1}+\epsilon}\left[H_{c}+\frac{\epsilon_{\text {eff }}}{T_{1}} \sum_{i} S_{i}^{x}+h_{\text {eff }} \sum_{i} S_{i}^{z}\right]+O(1 / \omega) .
\end{aligned}
$$

Note that $H_{\text {eff }}$ is a generic thermalizing Hamiltonian with no disorder and no MBL. Higher-order terms in $H_{\text {eff }}$ make it quasilocal with a decaying range of interactions. For the "unflipped" case when $\theta=\epsilon$, we consider a single period $U(T)$ (3) and the proportionality constant in $\hat{\mathcal{H}}_{F}^{(0)}$ is 1 , and $h_{\text {eff }}=h$ and $\epsilon_{\text {eff }}=\epsilon$. On the other hand, when $\theta=\pi+\epsilon$, we define $H_{\text {eff }}$ with respect to $U(2 T)$ the proportionality constant for $\hat{\mathcal{H}}_{F}^{(0)}$ is 2 , and the values $h_{\text {eff }}$ and $\epsilon_{\text {eff }}$ depend on $h$ and $\epsilon$ as discussed below.

With this in hand, we can predict drives for which prethermal-including time-crystalline-phenomena occur, and also understand the status of the experimental protocol in this regard. One of our main messages is that if $H_{\text {eff }}$ shows an approximate long-lived $\mathrm{U}(1)$ conservation, then $M(t)$ will saturate at a nonzero constant value on some (typically short) timescale under evolution with $H_{\text {eff }}$, for all initial states that start with a nonzero magnetization density. In the flipped case where $H_{\text {eff }}$ captures the dynamics over two periods (and hence over either even or odd times), a nonzero thermal value for $M(2 n T)$ implies period-doubled oscillations when considering both even and odd times because $P_{\pi}^{x} M P_{\pi}^{x}=-M$.

We will denote by $t_{m}$ the timescale on which the approximate $U(1)$ conservation is destroyed. This can arise via two mechanisms: (i) the system heats to the infinite temperature ensemble with no conservation laws, and $H_{\text {eff }}$ ceases to be a good description, which occurs on timescale $t_{h}$. Expectation values for all observables, including $M(t)$ become trivial at this time; (ii) the system thermalizes to the "true" equilibrium thermal ensemble for $H_{\text {eff }}$ which does not conserve $\mathrm{U}(1)$ symmetry (higher-order terms in $H_{\text {eff }}$ are not fine-tuned in any way, and will generally break the symmetry). At this time, denoted $t_{\text {th }}, M(t)$ will decay to zero for all initial states at infinite temperature with respect to $H_{\text {eff }}$, including those that started with a finite magnetization density. Note that $t_{\text {th }}$ will be set by a combination of $\epsilon$ and $\omega$ and could be made larger than $t_{h}$ for small-enough $\epsilon$. Thus, $t_{m} \sim \min \left[t_{\mathrm{th}}, t_{h}\right]$ will be set by the faster of the two processes above, and this sets the lifetime of the DTC response for the period-doubled case.

To examine the presence of $\mathrm{U}(1)$ conservation in an initial state independent manner, we consider the normalized quantity [27]

$$
\begin{aligned}
\Delta(n T) & \equiv \frac{1}{2 L}\|M(n T)-M(0)\|^{2} \\
& =\frac{1}{2 L} \frac{1}{2^{L}} \operatorname{Tr}\left\{[M(n T)-M(0)]^{\dagger}[M(n T)-M(0)]\right\} \\
& =1-\frac{1}{L} \frac{1}{2^{L}} \operatorname{Tr}[M(n T) M(0)] \\
& \equiv 1-C_{\text {tot }}(n T)
\end{aligned}
$$

Here, \|\| denotes the Hilbert-Schmidt operator norm, and $\Delta(n T)=0$ when $M$ is strictly conserved in which case $M(t)=M(0)$. The third line uses the fact that $\operatorname{Tr}\left[M^{\dagger}(t) M(t)\right]=\operatorname{Tr}[M(0) M(0)]=\sum_{i j} \operatorname{Tr}\left[\sigma_{i}^{z} \sigma_{j}^{z}\right]=L 2^{L}$ by the cyclicity of trace and the tracelessness of the Pauli operators. This expression relates the conservation of global magnetization to the infinite temperature autocorrelator of $M(t)$, which is experimentally measurable. We note that, more precisely, an approximate $\mathrm{U}(1)$ conservation will manifest itself as a dressed quasilocal operator $\tilde{M}$ that is conserved for a long-time $t_{m}$, and $\tilde{M}$ only agrees with $M$ to leading order in a small parameter [27].

We study the normalized autocorrelator $C_{\text {tot }}(t)$ defined above, and the deviation of the (absolute value) of this quantity from 1 is a proxy for the nonconservation of $M$ in the system. For an efficient numerical simulation of the system, we use quantum typicality [37-40] to replace the trace so that

$$
C_{\mathrm{tot}}(n T) \approx \frac{1}{L}\langle\tilde{\psi}|M(n T) M| \tilde{\psi}\rangle,
$$

where $|\tilde{\psi}\rangle$ is a random (Haar measure) state, typical for infinite temperature. We can then efficiently simulate the dynamics using numerically exact Krylov space-time evolution technique $[39,41,42]$ to calculate the action of matrix exponentials on wave functions. This allows us to access large systems of sizes $L=20-24$, beyond those accessible to exact diagonalization. Accessing these large sizes is particularly crucial in numerical studies of prethermalization which require us to work in the regime $J \ll \omega \ll J L$, where the first inequality is required to get a 
long-timescale $t_{h}$, and the second is required to keep the $O(1)$ frequency smaller than the extensive many-body bandwidth so as work in a sensible thermodynamic limit. In practice, the MB bandwidth is a factor of 5-10 larger than the frequency for the sizes we can achieve. The typicality approximation in Eq. (7) is exponentially accurate in terms of $L$, and for our Hilbert space dimensions $>10^{6}$, using a single wave function $|\tilde{\psi}\rangle$ is sufficient. We now examine the behavior of $C_{\text {tot }}(n T)$ for several different cases.

\section{A. Case 1: $h T_{1}=0$, Experimental Choice}

Let us start with the choice of parameters in the NMR experiment, in which a large Zeeman field is applied but the field is tuned so that $h T_{1}=0 \bmod 2 \pi$. This corresponds to $h_{\text {eff }}=0$ and $\epsilon_{\text {eff }}=\epsilon$ in $\hat{\mathcal{H}}_{F}^{(0)}$, Eq. (5). Note that one also obtains $h_{\text {eff }}=0$ if there is a $\pi$ pulse present, because the $z$ field flips sign and gets "echoed" out to leading order under the action of the $\pi$ flip. However, $h_{\text {eff }}=0$ is maximally nonideal from the point of view of the "usual" mechanism for generating an approximate U(1) conservation in a timeindependent Hamiltonian, which attempts to engineer a large separation of scales between different $S_{\text {tot }}^{z}$ sectors by subjecting the system a large magnetic field [27]. Indeed, the lifetime of TC response seen is the shortest for this case [Fig. 1(a)].

To wit, consider starting from an initial state that is at near-infinite temperature with respect to $H_{\text {eff }}$, but has a net magnetization density, similar to the experiment. Then, even if there is a long-lived $H_{\text {eff }}$ with $t_{h} \sim \exp (\omega / J)$, the appropriate thermal value for $M$ with respect to $H_{\text {eff }}$ is 0 , and $M(2 n T)$ will thermalize to zero on a timescale $t_{\text {th }}$ set by the destruction of $S_{\mathrm{tot}}^{z}$ conservation in $H_{\mathrm{eff}}$. In the absence of a large scale $h_{\text {eff }}$, this timescales as $t_{m} \sim t_{\text {th }} \sim 1 / \epsilon^{2}$ and depends polynomially on $1 / \epsilon$ by standard golden-rule-type reasoning.

In other words, even though $t_{h}$ scales exponentially with $\omega$, we do not expect $t_{m} \sim t_{\text {th }}$ to show a strong $\omega$ dependence. The $\omega$ independence is borne out by the numerical data in Figs. 1(a) and 2 and explains the relatively short lifetime of the time-crystalline response due to the absence of any exponential scaling in $t_{m}$. We note that since the actual experiment measures only 100 cycles, it still sees a finite Fourier peak at $\pi$ corresponding to the transient period doubling. Of course, in principle, one could also consider a regime with small-enough $\epsilon$ such that $t_{\text {th }}>t_{h}$. In this regime, the conservation of magnetization is destroyed due to $H_{\text {eff }}$ ceasing to be a good description rather than the destruction of $S_{\text {tot }}^{z}$ conservation within $H_{\text {eff }}$, i.e., $t_{m} \sim \min \left(t_{h}, t_{\mathrm{th}}\right)$; this corresponds to a window with a prethermal exponential dependence, $t_{m} \sim t_{h} \sim \exp (\omega)$ for the smallest range of $\epsilon$ 's.

A prethermal regime can be enhanced, however, through modifying the magnetic field $h$.

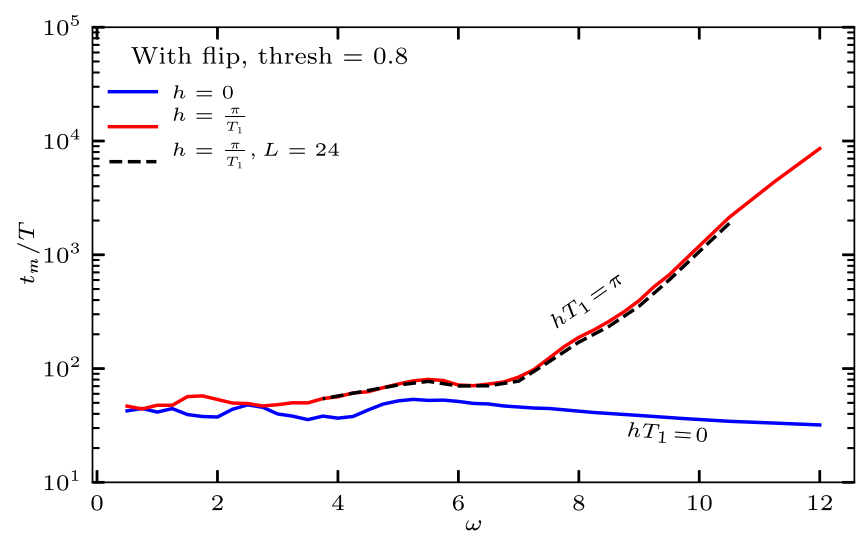

FIG. 2. Number of driving periods needed to reach a magnetization threshold of $C_{\text {tot }}=0.8$, which serves as an estimate for $t_{m}$ for the data in Fig. 1 with no $(h=0)$ and maximal field ( $h=\pi / T_{1}$ ). At large frequencies, the maximal field data shows a greatly increased $t_{m}$ with an exponential dependence on $\omega$. The dashed curves show additional data for longer chains $(L=24)$.

\section{B. Case 2: $h T_{1}=\pi / 2$, Generation of a prethermal regime}

Let us begin with the discussion of the nontrivial halfmaximal value (due to the compactness of the unit circle) $h T_{1}=\pi / 2$. Here, we consider the case without a $\pi$ pulse, i.e., we include only a small spin rotation by $\theta=\epsilon=0.1$ per period $T$ of the drive. This prevents the field from being "echoed out," so that $h_{\text {eff }}=h=\pi / 2 T_{1}$. Adding this field separates the different $S_{\text {tot }}^{z}$ sectors and makes the spin flip terms of strength $\epsilon$ more off-resonant and hence more ineffectual at destroying $S_{\text {tot }}^{z}$ conservation. However, as mentioned earlier, this field cannot be made parametrically large as is required for a bona fide prethermal U(1) regime in $H_{\text {eff }}$ (i.e., one with an exponentially long-lived in $h \mathrm{U}(1)$ conservation). Instead, it still the case that $S_{\text {tot }}^{z}$ conservation in $H_{\text {eff }}$ is destroyed on a polynomial in $1 / \epsilon$ timescale.

However, for this case, we show that prethermalization in the dynamics of $M(t)$ can be achieved by directly coupling to prethermalization of energy and relying on a notion of temperature, rather than relying on $\mathrm{U}(1)$ conservation. We note that the temperature can be quite high in this case, much higher than ordering temperatures for spontaneous symmetry breaking (in higher dimensions).

Consider again an initial state at a finite magnetization density. Now, due to the presence of the $z$ field in $H_{\text {eff }}$, this state is also at a finite energy density and hence temperature. Thus, $M(n T)$ will show an approximate "plateau" to a nonzero thermal value appropriate to the temperature of the initial state, before relaxing to zero at a later timescale $t_{m} \sim t_{h} \sim \exp [\omega / J]$, at which $H_{\text {eff }}$ ceases to be a good description.

Indeed, Fig. 1(b) shows an initial relaxation of $C_{\text {tot }}(n T)$ to a plateau at short times, followed by a later decay to zero as expected for an infinite temperature state with no additional conservation laws. We have verified that this 
later timescale scales as $t_{m} \sim \exp [\omega / J]$ (not shown). The dashed lines in the figure correspond to the thermal expectation value obtained by direct evolution with the time-averaged Hamiltonian $H_{\text {eff }}$, and these match the plateau values as expected.

\section{Case 3: $h T_{1}=\pi$, Prethermalization without temperature}

Finally, we consider the maximal possible field (again due to the compactness of the unit circle) of $h T_{1}=\pi$, which leads us to a demonstration of prethermalization without temperature, relying solely on $\mathrm{U}(1)$ conservation. We also return to the $\pi$-flip case to obtain a prethermal DTC. It turns out that for this value of $h$, the field segment of the drive also realizes a $\pi$ pulse, but now in the $z$ direction: $e^{-h T_{1} S_{\mathrm{tot}}^{z}} \sim P_{\pi}^{z}=\prod_{i} \sigma_{i}^{z}$. Again, let us look over two periods. Crucially, the effect of the $z \pi$ pulse is to "echo out" the $\epsilon S_{\text {tot }}^{x}$ term. Thus, $\hat{\mathcal{H}}_{F}^{(0)}$ over two periods has both $h_{\text {eff }}=0$ and $\epsilon_{\text {eff }}=0$ so that the (leading order) $H_{\text {eff }}$ exactly conserves $S_{\text {tot }}^{z}$.

Now, if one prepares initial states at infinite temperature with respect to $H_{\text {eff }}$ but finite magnetization density (which is possible because $h_{\text {eff }}=0$ ), then the initial value of $M$ will persist for the time that the dynamics is approximately governed by $H_{\text {eff. }}$ Then, at time $t_{m} \sim t_{h} \sim \exp (\omega / J)$, the magnetization decays to zero once $H_{\text {eff }}$ ceases to be a good description. As explained earlier, if one looks over both even and odd times, then oscillations are visible. This is confirmed in Fig. 1(c).

Put differently, when $H_{\text {eff }}$ has $\mathrm{U}(1)$ conservation, the equilibrium ensemble of $H_{\text {eff }}$ is characterized by both a temperature $\beta^{-1}$ and a chemical potential $\mu$. One can prepare initial states that have $\beta=0$, but have finite $\mu \neq 0$, and hence can show a persistent magnetization - thereby separating the notion of prethermalization from temperature by allowing for a separate thermodynamic parameter.

As is already visible by direct inspection of the time traces of $C_{\text {tot }}(n T)$ in Figs. 1(a) and 1(c), the lifetime of the approximate conservation of $S_{\text {tot }}^{z}$ and consequently the time-crystalline behavior is strongly enhanced by the presence of a magnetic field in the $z$ direction. In other words, a small modification of the applied field in the NMR experiment can lead to an exponentially greater DTC lifetime [43].

Figure 2 shows a direct comparison of the TC lifetimes in the case of zero and maximal field by extracting the time it takes for $C_{\text {tot }}(n T)$ to decay to a threshold value of 0.8 . At high driving frequencies $\omega \gtrsim 6$, we observe an exponential scaling of the lifetime with the frequency in the presence of the field - the characteristic signal of prethermalizationwhile without a field there is only a weak frequency dependence. The colored lines are extracted from the data for a chain of length $L=20$ in the other panels of 1 , while the black dashed line stems from the analysis of a larger system of size $L=24$. Note the negligibly small system-size dependence, which is to be expected as prethermalization is sensitive to the ratio of $O(1)$ parameter sizes rather than the system size.

Finally, we note that the exact conservation of $S_{\text {tot }}^{z}$ in $H_{\text {eff }}$ when $h T_{1}=\pi$ is only true to leading order in $1 / \omega$. Higherorder corrections at $O(\epsilon J / \omega)$ will again cause $S_{\text {tot }}^{z}$ to be destroyed within $H_{\text {eff }}$ on some polynomial timescale $t_{\text {th }} \sim \omega^{2} / \epsilon^{2}$. For very small $\epsilon$ 's and large $\omega$ 's such that $t_{\text {th }}<t_{h}$, we will find that $t_{m} \sim t_{\text {th }}$ does not show an exponential dependence on $\omega$. However, because the destruction of $S_{\text {tot }}^{z}$ conservation on timescales $t_{\text {th }}$ only occurs due to higher-order corrections in $H_{\text {eff }}$, in practice one can still isolate a large prethermal window where $t_{m} \sim t_{h} \sim \exp (\omega)$, as is visible from Fig. 1(c). In the limit that $\epsilon \rightarrow 0$, this window in $\omega$ can be made arbitrarily large.

Although discussed in the context of our model drive, the mechanism outlined above is very general. One can consider large families of drives for which the leading terms in $H_{\text {eff }}$ have a desired symmetry, with corrections only coming in at higher orders in $1 / \omega$. When the strength of these corrections is further controlled by a small parameter $\epsilon$, it is possible to tease out an exponentially large window in $\omega$ for small-enough $\epsilon$ during which the symmetry is approximately conserved.

We next turn to a more detailed verification of our picture, and its stability. First, Fig. 3 provides visually compelling direct evidence of the prethermal mechanism involving approximate $\mathrm{U}(1)$ conservation. It displays the participation entropy in the computational $z$ basis $\{|i\rangle\}$ upon starting from a specific basis state. The participation entropy of a wave function $|\psi\rangle$ in each magnetization sector $\mathcal{H}_{N_{\uparrow}}$ is defined as $S_{1}\left[N_{\uparrow}\right]=-\sum_{i:|i\rangle \in \mathcal{H}_{N_{\uparrow}}}|\langle i \mid \psi\rangle|^{2} \ln |\langle i \mid \psi\rangle|^{2}$, where $\mathcal{H}_{N_{\uparrow}}$ containing all basis states with $N_{\uparrow}$ spins pointing up.



FIG. 3. Time dependence of the sector (labeled by the number of up spins $N_{\uparrow}$ ) resolved participation entropy $S_{1}\left[|\psi(n T)\rangle, N_{\uparrow}\right]$ of the wave function $|\psi(n T)\rangle$ starting from the initial state $|00100100100100100100\rangle$ (i.e., in the sector with $N_{\uparrow}=6$ ) under the NMR Floquet drive with a frequency of $\omega=6.25, h T_{1}=\pi$, and an approximate global spin flip after each period $\theta=\pi+\epsilon$ with $\epsilon=0.18$. The wave function spreads quickly within one sector, before slowly spreading over several sectors. 

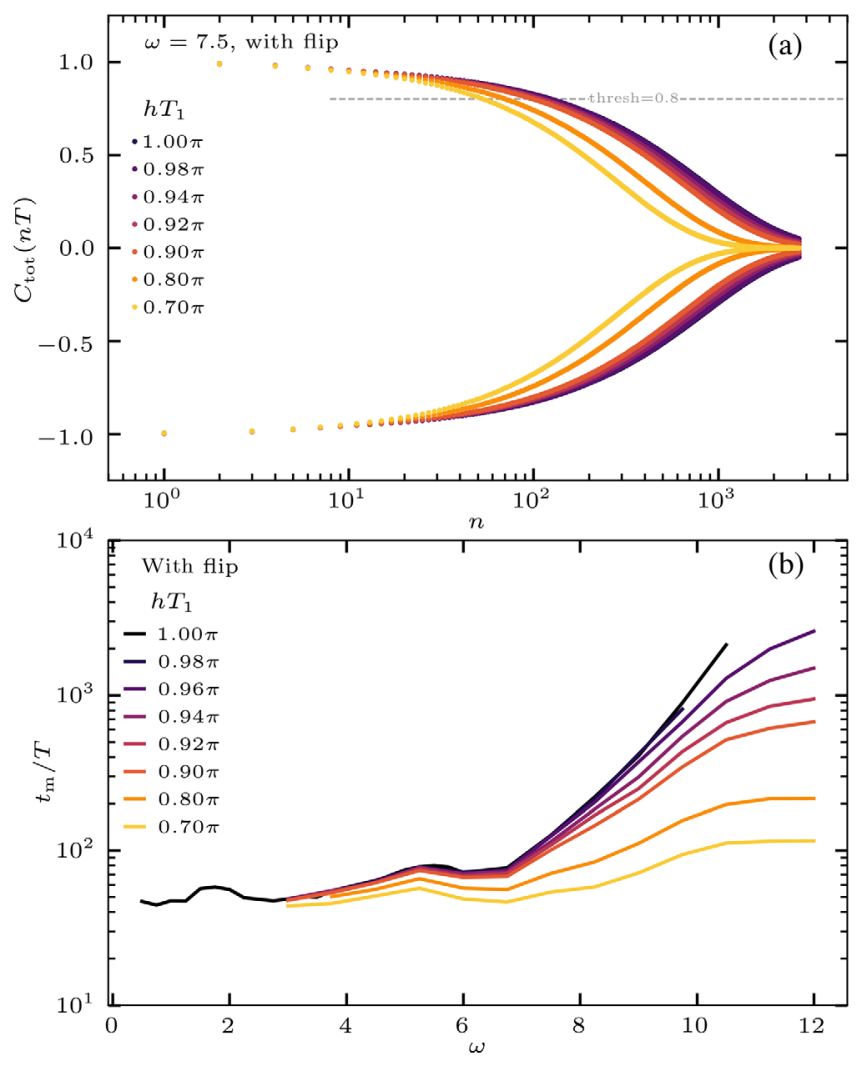

FIG. 4. Robustness of prethermal regime to drive imperfections. (a) Survival of the total magnetization $C_{\text {tot }}(n T)$ in a chain of length $L=20$ under the NMR Floquet drive (3) for driving frequency $2 \pi / T_{1}=7.5$ and magnetic fields $h$ detuned from the optimal field $h=\pi / T_{1}$. The detuning of the spin rotation $P_{\pi+\epsilon}^{x}$ is $\epsilon=0.1$. (b) Number of driving periods needed to reach a thresholds of 0.8 for the same data as in panel (a).

We find two distinct timescales: The wave function very quickly delocalizes in its initial magnetization sector, and then, much more slowly, leaks into increasingly distant other sectors, which is clearly visible in the delayed onset of the growth of the participation entropy for different magnetization sectors.

Finally, we note that a judiciously chosen $z$ field to realize a $\mathrm{U}(1)$ conservation in $H_{\text {eff }}$ is both experimentally feasible, and reminiscent of various "dynamical decoupling" schemes for Hamiltonian engineering. However, our results are not limited to a fine-tuned $z$ field. Figure 4 shows the stability of the prethermal DTC lifetimes to detuning from the maximal field. While for a maximal field the lifetimes are optimal as expected, the exponential scaling of the lifetimes with $\omega$ is still visible down to a detuning of about $10 \%$ from the maximal field.

\section{DISTINGUISHING BETWEEN PRETHERMAL AND MBL TCs}

For realistic experiments with a lifetime limited by extrinsic factors, it may often be the case that the prethermal time window is longer than the experimental lifetime. Thus, the question naturally arises of how to distinguish a prethermal DTC from a bona fide (MBLlocalized) infinitely long-lived one, and also how to distinguish between prethermal U(1) DTCs (this work) and prethermal SSB DTCs relying on spontaneous symmetry breaking (Ref. [30]). Indeed, all three DTC experiments thus far (on trapped ions, diamond NV centers, and NMR spins) nominally observe very similar experimental signatures, but for apparently different reasons.

To achieve this goal, we avail ourselves of the fundamentally distinct origin of the respective longevities. While the emergence of locally conserved quantities - the 1 bitsunderpin MBL [21,44,45], the prethermal U(1) DTC only offers a global conservation law which is not in conflict with local spin diffusion. The prethermal SSB DTC relies on yet a distinct mechanism which requires low-temperature initial states and spontaneous symmetry breaking.

The crispest way to distinguish between these mechanisms is by considering a variety of different initial states and measuring local spin autocorrelators in the $z$ basis. If we start with a random infinite temperature product state of $z$ spins in the $S_{\text {tot }}^{z}=0$ sector, only an MBL DTC will display oscillations in local autocorrelators $\left\langle\sigma_{i}^{z}(n T) \sigma_{i}^{z}\right\rangle$. These states are too high in temperature for prethermal SSB DTCs, and they have $S_{\mathrm{tot}}^{z}=0$ leading to zero net magnetization density for the U(1) DTC. Figure 5 displays a simulation of both local and global infinite temperature spin autocorrelators in the $z$ basis for the NMR model. In all settings displayed, we find that the local version decays much more quickly than the approximately symmetryprotected global one, while such a decay is precluded for the MBL version.

Finally, to distinguish between prethermal U(1) TCs and prethermal SSB TCs, one should start with initial states

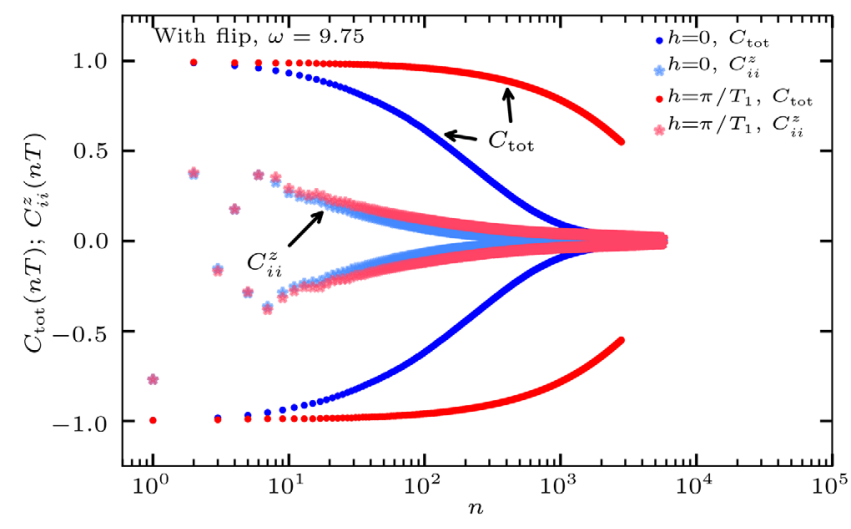

FIG. 5. Comparison between the survival of the global magnetization $C_{\text {tot }}(n T)$ and the local correlation function $C_{i i}^{z}=$ $\left(1 / 2^{L}\right) \operatorname{Tr}\left[Z_{i}(n T) Z_{i}\right]$. The former can decay much more slowly when there is an approximate global U(1) conservation, while the latter decays swiftly due to fast thermalization within $\mathrm{U}(1)$ sectors as shown in Fig. 3. This is in contrast to a many-body localized time crystal where both local and global autocorrelators oscillate with a finite amplitude even at infinitely late times. 
with $S_{\text {tot }}^{z}=0$, but still at a low temperature with respect to $H_{\text {eff }}$ (say, states with a single domain wall in the center of the chain). Local autocorrelators in the U(1) TC will thermalize within the $S_{\text {tot }}^{z}=0$ sector, showing no net magnetization and zero amplitude of oscillations. On the other hand, the prethermal SSB DTC will show oscillations in local correlators starting from such states, with occasional "phase slips" at late times due to slow coarsening dynamics of domain walls.

By contrast, if one starts from a polarized initial product state, then all three categories give virtually indistinguishable signatures. This is a drawback of existing TC experiments on disordered systems which only consider a very limited class of initial states. Indeed, the need for more finegrained experimental diagnostics was made particularly apparent in a recent theoretical study of the trapped ion experiment [5]. The trapped ion setup tries to realize an MBL DTC phase by engineering a Floquet Ising drive with imperfect $\pi$ flips, and disorder in the longitudinal $z$ fields. However, the disorder in the fields is echoed out under the $\pi$ flip to leading order, so that the model does not realize an MBL TC but rather looks to be a prethermal SSB DTC. If the experiment had been conducted for a wide variety of initial states (instead of only two low-temperature initial states) this difference would have been apparent.

Finally, one can ask if the mechanism of U(1) prethermalization might be at play in the trapped ion or NV center drives, since both models do have a U(1) symmetry for $\epsilon=0$. Similar to the NMR experiment, the NV experiment can only measure a global polarization, and the experiment starts with a fully polarized initial state and observes an oscillating signal for $M(t)$ with a slowly decaying envelope. Indeed, an effective Hamiltonian for this model is also obtained by adding a large $z$ field and going to an appropriate rotating frame-if this applied field were chosen to be $h T_{1}=\pi \bmod 2 \pi$ (which removes the deviation $\epsilon$ to leading order), then one would observe an enhancement of the DTC signal for this experiment as well. However, in the NV model, thermalization is a critically slow process due to disorder [35] — so that local autocorrelators would also decay slowly, unlike the NMR experiment where the decay of local correlators is fast. However, neither experiment has access to site-resolved local autocorrelations, so this difference between their thermalization mechanisms cannot be experimentally verified. The trapped ion experiment does measure local autocorrelators, but numerics for this model on different initial states are consistent with a prethermal SSB DTC rather than a prethermal U(1) DTC [1]. In principle, the trapped ion experiment could be repeated with a variety of different initial states to elucidate this difference.

\section{CONCLUSIONS}

In summary, we have analyzed in detail a scenario relevant for the optimization of NMR experiments on prethermal discrete time crystals in periodically driven quantum many-body systems. We argue that at highenough driving frequency, an optimal magnetic field exists which stabilizes an approximate $\mathrm{U}(1)$ conservation law and bears the potential to enhance the lifetimes of timecrystalline behavior by 2 orders of magnitude [Figs. 1(a) and 1(c)]. This optimization represents a small modification of the existing NMR experiment and should be achievable in practice.

One of our main contributions is to connect previous NMR insights with more rigorous theories of prethermalization to (i) demonstrate a large parameter window with an exponential lifetime for an emergent conservation law, even without large magnetic fields in $H_{\text {eff }}$, (ii) elucidate how this permits interesting prethermal dynamics, even at infinite temperature with respect to $H_{\text {eff }}$, and (iii) explain how these can be combined to obtain prethermal time crystals at high temperatures and without relying on the existence of symmetry breaking in $H_{\text {eff }}$. This, in turn opens up the possibility of realizing prethermal TCs in a much wider range of settings than is known thus far, because the need for SSB in thermalizing Hamiltonians comes with stringent constraints on allowed spatial dimensions and ranges of interactions due to Peierls-Mermin-Wagner-type theorems.

Finally, it is interesting to ask if the notion of an effective Hamiltonian can be dispensed with altogether to achieve drives with only a U(1) conservation without any notion of an effective Hamiltonian - the most dramatic rendition of prethermalization without temperature. Indeed, consider a drive with an oscillating magnetic field of the form [46]:

$$
U(T)=e^{-i(T / 2)\left(H_{c}+\epsilon S_{\mathrm{tot}}^{x}+h S_{\mathrm{tot}}^{z}\right)} e^{-i(T / 2)\left(H_{c}+\epsilon S_{\mathrm{tot}}^{x}-h S_{\mathrm{tot}}^{z}\right)} .
$$

In this case, the strength of the field $h$ can be made extremely large because of the presence of the noncommuting $\epsilon S_{\text {tot }}^{x}$ in both Hamiltonians. Now, if one works in the high-frequency limit, then the leading-order $H_{\text {eff }}$ averages over both terms and reduces to the $h_{\text {eff }}=0$ case considered earlier. On the other hand, in the low frequency limit where such an averaging is not appropriate and $H_{\text {eff }}$ is not defined, each term of the drive can be made to conserve $\mathrm{U}(1)$ in a crisp prethermal sense, thereby endowing $\mathrm{U}(1)$ conservation to the drive as a whole. We have qualitatively verified numerically that this drive has enhanced $M(t)$ conservation at small rather than large frequencies. However, obtaining a quantitative agreement is limited by finite-size numerics due to our inability to access a regime where $J \ll h \ll \omega \ll J L$.

\section{ACKNOWLEDGMENTS}

We thank Sean Barrett, Robert Blum, Jared Rovny, Sarang Gopalakrishnan, and Wen Wei Ho for discussions. R. M. thanks Arnab Das, Asmi Haldar, and Diptiman Sen for collaboration on related topics. V. K. and S. L. S. thank Curt von Keyserlingk and Matteo Ippoliti for collaboration 
on related topics. This work was in part supported by the DFG through ct.qmat (EXC 2147, Project No. 39085490) and SFB 1143 (Project No. 247310070). V. K. was supported in part by the Harvard Society of Fellows and the William F. Milton Fund. This research was also developed with funding from the Defense Advanced Research Projects Agency (DARPA) via the DRINQS program. D. J. L. thanks PRACE for awarding access to HLRS's Hazel Hen computer based in Stuttgart, Germany under Grant No. 2016153659. The views, opinions, and/or findings expressed are those of the authors and should not be interpreted as representing the official views or policies of the Department of Defense or the U.S. Government. Our code is based on the PETSc and SLEPc libraries.

[1] V. Khemani, A. Lazarides, R. Moessner, and S. L. Sondhi, Phase Structure of Driven Quantum Systems, Phys. Rev. Lett. 116, 250401 (2016).

[2] D. V. Else, B. Bauer, and C. Nayak, Floquet Time Crystals, Phys. Rev. Lett. 117, 090402 (2016).

[3] C. W. von Keyserlingk, V. Khemani, and S. L. Sondhi, Absolute Stability and Spatiotemporal Long-Range Order in Floquet Systems, Phys. Rev. B 94, 085112 (2016).

[4] R. Moessner and S. L. Sondhi, Equilibration and Order in Quantum Floquet Matter, Nat. Phys. 13, 424 (2017).

[5] V. Khemani, R. Moessner, and S. L. Sondhi, A Brief History of Time Crystals, arXiv:1910.10745.

[6] D. V. Else, C. Monroe, C. Nayak, and N. Y. Yao, Discrete Time Crystals, arXiv:1905.13232.

[7] K. Sacha and J. Zakrzewski, Time Crystals: A Review, Rep. Prog. Phys. 81, 016401 (2018).

[8] L. D'Alessio and M. Rigol, Long-Time Behavior of Isolated Periodically Driven Interacting Lattice Systems, Phys. Rev. X 4, 041048 (2014).

[9] A. Lazarides, A. Das, and R. Moessner, Equilibrium States of Generic Quantum Systems Subject to Periodic Driving, Phys. Rev. E 90, 012110 (2014).

[10] P. Ponte, A. Chandran, Z. Papic, and D. A. Abanin, Periodically Driven Ergodic and Many-Body Localized Quantum Systems, Ann. Phys. (Amsterdam) 353, 196 (2015).

[11] A. Lazarides, A. Das, and R. Moessner, Fate of Many-Body Localization under Periodic Driving, Phys. Rev. Lett. 115, 030402 (2015).

[12] P. Ponte, Z. Papić, F. Huveneers, and D. A. Abanin, ManyBody Localization in Periodically Driven Systems, Phys. Rev. Lett. 114, 140401 (2015).

[13] D. A. Abanin, W. De Roeck, and F. Huveneers, Theory of Many-Body Localization in Periodically Driven Systems, Ann. Phys. (Amsterdam) 372, 1 (2016).

[14] P. W. Anderson, Absence of Diffusion in Certain Random Lattices, Phys. Rev. 109, 1492 (1958).

[15] D. M. Basko, I. L. Aleiner, and B. L. Altshuler, MetalInsulator Transition in a Weakly Interacting Many-Electron System with Localized Single-Particle States, Ann. Phys. (Amsterdam) 321, 1126 (2006).
[16] I. V. Gornyi, A. D. Mirlin, and D. G. Polyakov, Interacting Electrons in Disordered Wires: Anderson Localization and Low-T Transport, Phys. Rev. Lett. 95, 206603 (2005).

[17] A. Pal and D. A. Huse, Many-Body Localization Phase Transition, Phys. Rev. B 82, 174411 (2010).

[18] M. Žnidarič, T. Prosen, and P. Prelovšek, Many-Body Localization in the Heisenberg XXZ Magnet in a Random Field, Phys. Rev. B 77, 064426 (2008).

[19] V. Oganesyan and D. A. Huse, Localization of Interacting Fermions at High Temperature, Phys. Rev. B 75, 155111 (2007).

[20] D. J. Luitz, N. Laflorencie, and F. Alet, Many-Body Localization Edge in the Random-Field Heisenberg Chain, Phys. Rev. B 91, 081103(R) (2015).

[21] J. Z. Imbrie, On Many-Body Localization for Quantum Spin Chains, J. Stat. Phys. 163, 998 (2016).

[22] R. Nandkishore and D. A. Huse, Many-Body Localization and Thermalization in Quantum Statistical Mechanics, Annu. Rev. Condens. Matter Phys. 6, 15 (2015).

[23] D. A. Abanin, E. Altman, I. Bloch, and M. Serbyn, Colloquium: Many-Body Localization, Thermalization, and Entanglement, Rev. Mod. Phys. 91, 021001 (2019).

[24] V. Khemani, C. W. von Keyserlingk, and S. L. Sondhi, Defining Time Crystals via Representation Theory, Phys. Rev. B 96, 115127 (2017).

[25] D. A. Abanin, W. De Roeck, and F. Huveneers, Exponentially Slow Heating in Periodically Driven Many-Body Systems, Phys. Rev. Lett. 115, 256803 (2015).

[26] D. A. Abanin, W. De Roeck, W. W. Ho, and F. Huveneers, Effective Hamiltonians, Prethermalization, and Slow Energy Absorption in Periodically Driven Many-Body Systems, Phys. Rev. B 95, 014112 (2017).

[27] D. Abanin, W. De Roeck, W. W. Ho, and F. Huveneers, A Rigorous Theory of Many-Body Prethermalization for Periodically Driven and Closed Quantum Systems, Commun. Math. Phys. 354, 809 (2017).

[28] T. Mori, T. Kuwahara, and K. Saito, Rigorous Bound on Energy Absorption and Generic Relaxation in Periodically Driven Quantum Systems, Phys. Rev. Lett. 116, 120401 (2016).

[29] T. Kuwahara, T. Mori, and K. Saito, Floquet-Magnus Theory and Generic Transient Dynamics in Periodically Driven Many-Body Quantum Systems, Ann. Phys. (Amsterdam) 367, 96 (2016).

[30] D. V. Else, B. Bauer, and C. Nayak, Prethermal Phases of Matter Protected by Time-Translation Symmetry, Phys. Rev. X 7, 011026 (2017).

[31] J. Rovny, R. L. Blum, and S. E. Barrett, Observation of Discrete-Time-Crystal Signatures in an Ordered Dipolar Many-Body System, Phys. Rev. Lett. 120, 180603 (2018).

[32] J. Rovny, R. L. Blum, and S. E. Barrett, ${ }^{31} \mathrm{P}$ NMR Study of Discrete Time-Crystalline Signatures in an Ordered Crystal of Ammonium Dihydrogen Phosphate, Phys. Rev. B 97, 184301 (2018).

[33] S. Choi, J. Choi, R. Landig, G. Kucsko, H. Zhou, J. Isoya, F. Jelezko, S. Onoda, H. Sumiya, V. Khemani, C. von Keyserlingk, N. Y. Yao, E. Demler, and M. D. Lukin, Observation of Discrete Time-Crystalline Order in a Disordered Dipolar Many-Body System, Nature (London) 543, 221 (2017). 
[34] J. Zhang, P. W. Hess, A. Kyprianidis, P. Becker, A. Lee, J. Smith, G. Pagano, I.-D. Potirniche, A. C. Potter, A. Vishwanath, N. Y. Yao, and C. Monroe, Observation of a Discrete Time Crystal, Nature (London) 543, 217 (2017).

[35] W. W. Ho, S. Choi, M. D. Lukin, and D. A. Abanin, Critical Time Crystals in Dipolar Systems, Phys. Rev. Lett. 119, 010602 (2017).

[36] J. Choi, H. Zhou, S. Choi, R. Landig, W. W. Ho, J. Isoya, F. Jelezko, S. Onoda, H. Sumiya, D. A. Abanin, and M.D. Lukin, Probing Quantum Thermalization of a Disordered Dipolar Spin Ensemble with Discrete Time-Crystalline Order, Phys. Rev. Lett. 122, 043603 (2019).

[37] C. Bartsch and J. Gemmer, Dynamical Typicality of Quantum Expectation Values, Phys. Rev. Lett. 102, 110403 (2009).

[38] P. Reimann, Dynamical Typicality of Isolated ManyBody Quantum Systems, Phys. Rev. E 97, 062129 (2018).

[39] D. J. Luitz and Y. B. Lev, The Ergodic Side of the ManyBody Localization Transition, Ann. Phys. (Berlin) 529, 1600350 (2017).
[40] D. J. Luitz, Y. B. Lev, and A. Lazarides, Absence of Dynamical Localization in Interacting Driven Systems, SciPost Phys. 3, 029 (2017).

[41] A. Nauts and R. E. Wyatt, New Approach to Many-State Quantum Dynamics: The Recursive-Residue-Generation Method, Phys. Rev. Lett. 51, 2238 (1983).

[42] C. Moler and C. Van Loan, Nineteen Dubious Ways to Compute the Exponential of a Matrix, Twenty-Five Years Later, SIAM Rev. 45, 3 (2003).

[43] Note that a large magnetic field is used in the NMR setup to obtain an interaction Hamiltonian $H_{c}$ that conserves $S_{\text {tot }}^{z}$ within the secular approximation. However, the periodic drive as a whole comprises both the interaction Hamiltonian (with the field) and the global spin rotation, and there is no simple large field limit to obtain $S_{\text {tot }}^{z}$ conservation for $\mathrm{U}(T)$ as a whole.

[44] D. A. Huse, R. Nandkishore, and V. Oganesyan, Phenomenology of Fully Many-Body-Localized Systems, Phys. Rev. B 90, 174202 (2014).

[45] M. Serbyn, Z. Papić, and D. A. Abanin, Local Conservation Laws and the Structure of the Many-Body Localized States, Phys. Rev. Lett. 111, 127201 (2013).

[46] A. Haldar, R. Moessner, and A. Das, Onset of Floquet Thermalization, Phys. Rev. B 97, 245122 (2018). 